Entzündliche Gelenkschmerzen

\title{
Gute Beweglichkeit - weniger gastrointestinale Komplikationen
}

- Firstline-Medikamente zur Therapie von Gelenkschmerzen und Steifigkeit sind nichtsteroidale entzündungshemmende Wirkstoffe wie traditionelle NSAR (tNSAR) und Coxibe, führte PD Dr. Michael Überall, Nürnberg aus. Obwohl das gastrointestinale Risiko von tNSAR seit langem bekannt ist, würden gerade ältere Patienten, die per se oft Kontraindikationen für NSAR hätten, damit behandelt. Das Risiko für Ulzerationen und Blutungen sei besonders in den ersten vier Wochen der Anwendung hoch und könne damit auch nicht durch eine Kurzzeittherapie umgangen werden, so Überall.

\section{Mukosaschutz weitgehend erhalten}

Coxibe wie das kürzlich in das SchmerzPortfolio von Grünenthal aufgenommene Etoricoxib (Exinef ${ }^{\oplus}$ ) beeinflussen im Gegensatz zu tNSAR wie Diclofenac und Ibuprofen die gastrale Mukosaprotektion deutlich weniger, was sich positiv auf die gastrointestinale Verträglichkeit auswirkt. Zudem bewirken Coxibe keine Plättchenaggrega- tionshemmung, die bei den tNSAR das Blutungsrisiko steigert [Baraf HSB et al. J Rheumatol 2007;34:408-420; Hunt RH et al., Am J Gastroenterol 2003,98:1725-1733].

Unter Etoricoxib $(1 \times 120 \mathrm{mg} / \mathrm{d})$ ist die kumulative Häufigkeit gastrointestinaler Ulzerationen deutlich geringer als unter Ibuprofen $(3 \times 800 \mathrm{mg} / \mathrm{d})$, wie eine randomisierte Doppelblindstudie mit 680 Teilnehmern gezeigt hat [Hunt $\mathrm{RH}$ et al. Am J Gastroenterol 2003;98:1725-1733]. Klinische Studien bei Patienten mit Knieoder Hüftarthrose belegen zudem, dass Etoricoxib die Arthroseschmerzen auf dem WOMAC-Index für Gelenkschmerz sowie die Mobilität (WOMAC-Subskala körperliche Funktion) über einen Zeitraum von mehr als einem Jahr deutlich verbessern kann [Bingham $\mathrm{CO}$ et al. Rheumatology 2007;46:496].

- Dagmar Jäger-Becker

Quelle: Einführungspressekonferenz „EXINEF ${ }^{\oplus}$

- für ein Mehr an Bewegung bei Gelenk-

schmerzen"; Frankfurt, April 2013 (Veranstalter: Grünenthal)
Kurz notiert

Psoriasis-Broschüre von Springer Medizin

- Die Patientenbroschüre „20 Fragen - 20 Antworten“, die mit Unterstützung von LEO Pharma realisiert worden ist, beantwortet auf knapp 30 Seiten die häufigsten Fragen, die im Zusammenhang mit der Erkrankung, ihrer Therapie und den psychosozialen Auswirkungen immer wieder in der Praxis gestellt werden. Woher kommt die Erkrankung? Wie kann ich sie durch die richtige Lebensführung günstig beeinflussen? Welche Therapiemöglichkeiten gibt es? Wo finde ich zusätzliche Informationen? Bestellungen per E-Mail an: vertrieb@springer.com oder per Fax an 06102/506240, Stichwort „20 Fragen - Psoriasis".

- Volker Schuck, Springer Medizin

\section{Jod- und Selenversorgung in Deutschland}

\section{Noch kein Grund zur Entwarnung}

- Obwohl die WHO Deutschland von der Liste der Länder mit Jodmangel gestrichen hat (es liegt an der unteren Grenze der von der WHO definierten ausreichenden Jodversorgung von 100-200 $\mu \mathrm{g} / \mathrm{l}$ ), besteht kein Grund zur Entwarnung.

\section{Jodversorgung bei Kindern}

Um die Jodversorgung auf Bevölkerungsebene zu beurteilen, hat das Robert KochInstitut im Jodmonitoring des Kinder- und Jugendsurveys (KiGGS) 2007 deutschlandweit bei 14134 Kindern im Alter von 0-17 Jahren die Jodausscheidung im Urin gemessen. Die mediane Jodausscheidung betrug $117 \mu \mathrm{g} / \mathrm{l}$. Allerdings hatten 40\% der Kinder eine Jodversorgung, die unter 100 $\mu \mathrm{g} / \mathrm{l}$ lag und $17 \%$ der Kinder sogar eine un- ter $50 \mu \mathrm{g} / \mathrm{l}$. Eine Nachuntersuchung hat jedoch gezeigt, dass Kinder und Jugendliche noch ganz gut versorgt waren.

Deutliche Versorgungslücken finden sich eher bei Schwangeren. "Sie brauchen Jod - vom ersten Tag der Schwangerschaft bis über die Stillzeit hinaus." betont PD Dr. Joachim Feldkamp, Bielefeld.

Da Tierfutter jodiert wird, erhält die deutsche Bevölkerung einen Großteil des Jodbedarfs aus Milchprodukten (37\%) und Fleischprodukten (21\%). Durch die Empfehlung, den Salzkonsum zu verringern, und den niedrigeren Verbrauch an jodiertem Salz in der Lebensmittelindustrie wird eventuell eine Anpassung der Höhe der Jodbeimischung zum Speisesalz notwendig, fasst Feldkamp zusammen.

\section{Selen vor allem im Eiweiß}

Die Selenversorgung in Deutschland scheint derzeit ausreichend. Der physiologische Selenspiegel liegt bei 50-120 $\mu \mathrm{g} / \mathrm{l}$. Es wird empfohlen, täglich 35-70 $\mu \mathrm{g}$ aufzunehmen. Mit $65 \%$ ist tierisches Eiweiß die Hauptquelle unserer Seelenzufuhr. Feldkamp präsentiert Daten, die zeigen, dass die Selenwerte umso schlechter sind, je weniger Fleisch- und tierische Produkte die Untersuchten zu sich genommen haben.

- Ulrike Tietze

Quelle: Pressekonferenz „Status Quo zur Versorgungslage mit Jod und Selen in Deutschland" im Rahmen 100 Jahre Henning - Schilddrüsensymposium, Berlin, April 2013 (Veranstalter: Sanofi-Aventis Deutschland GmbH / Henning) 